\title{
Depressive Symptom Trajectory Predicts One-Year Health- Related Quality of Life in Patients with Heart Failure
}

\author{
Rebecca L. Dekker, PhD, APRN ${ }^{1}$, Terry A. Lennie, PhD, RN ${ }^{1}$, Nancy M. Albert, PhD, CCNS ${ }^{2}$, \\ Mary K. Rayens, $\mathrm{PhD}^{1}$, Misook L. Chung, PhD, RN ${ }^{1}$, Jia-Rong $\mathrm{Wu}, \mathrm{PhD}, \mathrm{RN}^{3}$, Eun Kyeung \\ Song, PhD, $\mathbf{R N}^{6}$, and Debra K. Moser, DNSc, RN ${ }^{1}$ \\ ${ }^{1}$ University of Kentucky College of Nursing \\ ${ }^{2}$ Cleveland Clinic, Cleveland, $\mathrm{OH}$ \\ ${ }^{3}$ University of North Carolina School of Nursing, Chapel Hill \\ ${ }^{6}$ University of Ulsan Department of Nursing, South Korea
}

\begin{abstract}
Background-One-third of patients with heart failure (HF) experience depressive symptoms that adversely affect health-related quality of life (HRQOL). We aimed to describe depressive symptom trajectory and determine whether a change in depressive symptoms predicts subsequent HRQOL.
\end{abstract}

Methods and Results-The sample consisted of 256 inpatients and outpatients with HF. Depressive symptoms were measured at baseline and 3 or 6 months with the Patient Health Questionnaire (PHQ-9). The Minnesota Living with HF Questionnaire was used to assess HRQOL at baseline, 3-6 months, and one year. Based on baseline and 3-6 month PHQ-9 scores, patients were categorized as depressive symptom-free (64\%), depressive symptoms improved (15\%), depressive symptoms developed (6\%), or persistent depressive symptoms (15\%). The groups differed in one-year HRQOL levels $(\mathrm{F}=36, p<.001)$; patients who were depressive symptom-free or whose depressive symptoms improved had better one-year HRQOL than patients with persistent depressive symptoms (Tukey HSD, $p<.01$ ). Change in depressive symptoms was the strongest predictor of one-year HRQOL (standardized $\beta=.42, p<.001$ ), after controlling for functional status, demographics, and clinical variables.

Conclusions-We found the trajectory of depressive symptoms predicts future HRQOL. Research is needed to determine whether interventions targeting depressive symptoms improve HRQOL in patients with HF.

\section{Keywords}

Cardiovascular; Depression

() 2011 Elsevier Inc. All rights reserved.

Corresponding author: Rebecca L. Dekker, PhD, APRN, ACNS-BC, Assistant Professor, University of Kentucky College of Nursing, 760 Rose Street, Lexington, KY 40536-0232, Office: 859-323-0242; FAX: 859-323-1057, rdekker@uky.edu.

Publisher's Disclaimer: This is a PDF file of an unedited manuscript that has been accepted for publication. As a service to our customers we are providing this early version of the manuscript. The manuscript will undergo copyediting, typesetting, and review of the resulting proof before it is published in its final citable form. Please note that during the production process errors may be discovered which could affect the content, and all legal disclaimers that apply to the journal pertain.

Disclosures

The authors have nothing to disclose. 


\section{Introduction}

One-third of all patients with heart failure (HF) experience clinically significant depressive symptoms ${ }^{1}$ that adversely affect every aspect of health-related quality of life (HRQOL). ${ }^{2}$ Health-related quality of life is a subjective, patient-centered outcome that refers to how a health condition affects a person's total well-being, including physical, functional, and social dimensions. ${ }^{3}$ Health-related quality of life is an important predictor of morbidity and mortality in patients with $\mathrm{HF}^{4,5}$ Researchers have also begun to view HRQOL as an important endpoint of HF care, given that patients with $\mathrm{HF}$ are experiencing longer survival and must adjust to life with a chronic condition. ${ }^{6}$

It has been hypothesized that interventions aimed at reducing depressive symptoms in patients with HF may lead to an improvement in health outcomes, including HRQOL. ${ }^{2}$ This hypothesis is based on results from multiple cross-sectional studies showing that depressive symptoms measured at baseline independently predict baseline HRQOL in patients with HF. ${ }^{2,7-10}$ Although few investigators have examined the natural trajectory of depressive symptoms in patients with $\mathrm{HF},{ }^{11-14}$ the severity of depressive symptoms may vary over time. It is not known whether a positive or negative change in depressive symptoms over time leads to a parallel change in HRQOL.

Therefore, the primary purpose of this study was to determine whether a change in depressive symptom status from baseline to 3-6 months predicts HRQOL at one year. A secondary aim was to examine the trajectory of depressive symptoms and HRQOL in patients with HF. We hypothesized that 1) there would be a significant group by time effect for depressive symptom status and the trajectory of depressive symptoms at baseline and 36 months, 2) there would be a significant group by time effect for depressive symptom status and HRQOL at baseline, 3-6 months, and one year; 3) patients who were depressive symptom-free or whose depressive symptoms improved would have better HRQOL at one year compared to patients with persistent depressive symptoms or those who developed depressive symptoms; and 4) depressive symptom status would independently predict subsequent HRQOL at one year.

\section{Conceptual Framework}

A model presented by Kop, Synowski, and Gottlieb ${ }^{15}$ provides the conceptual framework for our study. In their model, HF symptoms, such as dyspnea, fatigue, and exercise intolerance, influence both depressive symptoms and functional limitations. Depressive symptoms and functional limitations are co-related, and both of these factors contribute to impaired HRQOL. Although HRQOL is influenced primarily by subjective factors, such as depressive symptoms and functional limitations, the construct of HRQOL is thought to represent a composite of multiple influences, and is more than the simple sum of the parts. HRQOL represents the patient's global perspective of their daily life given the many physical health, emotional, social, economic and health perception factors affecting it. Thus although depressive symptoms and functional status may influence HRQOL, these are not equivalent to HRQOL, nor can HRQOL be conceptualized simply as existing of $30 \%$ depression, $30 \%$ symptom status, and so on.

Unfortunately, existing models of HRQOL in HF are based primarily on findings from cross-sectional studies. In their discussion of HRQOL in HF, Kop et al. ${ }^{15}$ state there is a need for future researchers to conduct longitudinal studies to advance the state of the science. In conducting our study, we aimed to contribute to the knowledge gap on the longitudinal relationship between depressive symptoms and HRQOL in patients with HF. 


\section{Methods}

\section{Design and Sample}

We used a longitudinal design with repeated measures. This was a secondary data analysis of data from the Heart Failure Quality of Life registry for patients with HF. This registry is a joint venture between investigators at 8 different medical centers; a detailed summary of the methods has been previously published. ${ }^{16,17}$ The present subset $(\mathrm{N}=256)$ includes a convenience sample of subjects who had complete data on depressive symptoms at baseline and 3 or 6 months, and HRQOL at one year. Of registry sites, two collected variables at the desired time points.

The first study site was in acute care (hospital setting) at the main campus of Cleveland Clinic in Ohio. The purpose of the longitudinal, observational study at this site was to describe the trajectory of HRQOL in hospitalized patients with HF. Patients in this study received usual care. The second study site was the outpatient setting of an academic medical center in Kentucky. The purpose of the study at the second site was to test the effects of combined biofeedback and cognitive therapy on HF outcomes (National Institutes of Health/ National Institutes of Nursing Research R01 NR 008567). Patients were recruited in the outpatient setting and randomized to usual care, attention placebo, or an intervention group.

Both studies used similar inclusion and exclusion criteria. Patients were eligible for inclusion if they had a diagnosis of chronic HF, preserved or non-preserved systolic function, and were English-speaking. They were excluded for a myocardial infarction within 3 months or unstable angina, cognitive impairment, discharge to a skilled nursing facility, or severe psychiatric impairment other than depression or anxiety. Patients recruited in the outpatient setting were screened for one additional inclusion criteria - these patients were included only if they had been optimized on HF medications and on stable doses for three months.

\section{Protocol}

Local institutional review boards approved the individual studies and the review board at the primary author's institution approved secondary data analysis as an exempt protocol. Patients recruited in the acute care setting provided informed consent and participated in a baseline assessment during hospitalization. Follow-up measurement of depressive symptoms and HRQOL took place by phone or mail at six months and one year. In the second study setting, patients completed informed consent and baseline, three-month, and one-year assessments at a General Clinical Research Center. After completion of each study, data were de-identified and integrated into a single database at the primary author's institution.

\section{Measurement}

Depressive symptoms-Depressive symptoms were defined as symptoms of depression that may exist or without a diagnosis of major depressive disorder. ${ }^{18}$ Somatic symptoms of depression may overlap with symptoms of HF such as fatigue or changes in appetite. ${ }^{19}$ However, we chose to categorize all patients with clinically significant depressive symptoms as having depressive symptoms, regardless of whether or not the somatic symptoms could be related to HF. This approach is known as the inclusive approach to measuring depressive symptoms. ${ }^{20}$ Researchers have provided strong evidence that the inclusive approach is sensitive, specific reliable, and predicts persistent depressive symptoms in patients who are physically ill. ${ }^{21,22}$

The Patient Health Questionnaire, or PHQ-9, was used to measure depressive symptoms. This is a 9-item questionnaire based directly on the diagnostic criteria for major depressive 
disorder. ${ }^{23}$ Patients are asked to rate how often each symptom has bothered them during the past 2 weeks on a scale from 0 (not at all) to 3 (nearly every day). Patients who score a 10 or greater are considered to have moderate or greater depressive symptoms. The PHQ-9 is an ideal instrument for measuring depressive symptoms because it is brief, responsive to change over time, and has strong evidence for reliability and validity in medical outpatients $^{24}$ and patients with HF. ${ }^{25}$ The Cronbach's alpha for our sample was .83 , providing evidence of adequate reliability.

Health-related quality of life-The Minnesota Living with Heart Failure questionnaire is a HF-specific measure of HRQOL that assesses the patient's perceptions of the influence of HF on physical, socioeconomic, and emotional aspects of life. ${ }^{26}$ The 21 items are summed with higher scores indicating worse HRQOL. The Minnesota Living with HF questionnaire is one of the most widely used instruments for measuring quality of life in this population. Researchers have demonstrated evidence for validity and reliability. ${ }^{27}$ The Cronbach's alpha in our study was 0.92 , adding support for reliability.

Functional status-We measured baseline subjective functional status using the Duke Activity Status Index (DASI) ${ }^{28}$ and New York Heart Association (NYHA) functional class. ${ }^{29}$ The DASI is a 12 -item questionnaire that assesses the functional capacity for activities of daily living. Patients are asked whether they can perform certain activities, such as walking a block or two on level ground. There are three response categories: 'not done because of health reasons', 'done with difficulty', and 'done without difficulty.' Total scores are calculated based on a weighted score for each activity; higher total scores indicate better functional status. New York Heart Association functional class is a subjective indicator of functional status and was determined by patient interview. Patients were assigned a classification of I (ordinary physical activity causes no symptoms of fatigue, dyspnea, angina or palpitations), II (symptoms with ordinary physical activity), III (symptoms occur with less than ordinary physical activity) or IV (symptoms occur even at rest).

Demographics and clinical characteristics-To completely describe the sample and obtain data on potential confounding variables, the following information was collected by patient interview and chart review: age, sex, race/ethnicity, marital status, whether the patient lives alone, education level, and time since diagnosis. The following clinical characteristics were collected by chart review: height, weight, smoking status, most recent ejection fraction, and medications. Data on comorbidites were collected by chart review and patient interview using the Charlson Comorbidity Index. ${ }^{30,31}$

\section{Data analysis}

SPSS version 18.0 was used for data analysis; a $p$ value of less than .05 was considered significant. Patient characteristics were summarized using means and standard deviations or frequency distributions. Differences in demographic and clinical variables between the two study sites were assessed with independent t-tests or chi-square tests of association.

The standard PHQ-9 cut-point of 10 was used to categorize patients as having depressive symptoms or no symptoms at baseline and 3-6 months. We then placed patients into one of four depressive symptom status groups based on change in PHQ-9 score or the lack of PHQ-9 change from baseline to 3-6 months (See Table 1). These 4 groups were: 1) depressive symptom-free, 2) depressive symptoms improved, 3) depressive symptoms developed, and 4) persistent depressive symptoms. To assess the trajectory of depressive symptoms among these four groups, repeated measures analysis of variance with Tukey honestly significant difference (HSD) post-hoc tests were used. 
Repeated measures and one-way analysis of variance with Tukey HSD post-hoc tests were used to determine the relationship between depressive symptom status and HRQOL.

Multiple regression analysis was used to determine whether a change in depressive symptom status predicted HRQOL at one year, controlling for demographics, clinical variables, and functional status. For the regression, patients in each depressive symptom status group were assigned a dummy variable ranked from best to worst: (0) depressive symptom-free, (1) depressive symptoms improved, (2) depressive symptoms developed, or (3) persistent depressive symptoms. To account for differences between the two sites, we controlled for recruitment setting and treatment group (usual care, attention placebo, or intervention) in the final regression model.

\section{Results}

\section{Sample Characteristics}

The final sample consisted of 256 patients, one-third of who were female. Approximately half of the sample was classified as NYHA functional status class III or IV. In Table 2, the baseline characteristics of patients recruited in the acute care $(n=108)$ and outpatient settings $(n=148)$ were compared. There was a higher percentage of patients with NYHA class III among patients from the acute care setting $(p=.003)$. Patients from the acute care setting also had poorer functional status as measured by the DASI.

There were a higher percentage of patients from the acute care setting with a history of coronary artery bypass surgery ( $41 \%$ vs. $24 \%, p=.003)$. Patients from the acute care setting had a lower mean ejection fraction, lower body mass index, and fewer years of education compared to patients from the outpatient setting. There were no data available on medications for the patients from the acute care setting. Among outpatients, $23 \%$ were taking antidepressants, and $85 \%$ were taking an ACE inhibitor or an angiotensin receptor blocker. No other demographic and clinical characteristic differed between the two groups.

\section{Trajectory of Depressive Symptoms}

From baseline to 3-6 months, a total of 164 patients (64\%) remained depressive symptomfree, while $39(15 \%)$ experienced improvement in depressive symptoms, $14(6 \%)$ developed depressive symptoms, and $39(15 \%)$ had persistent depressive symptoms. A higher percentage of patients recruited from the outpatient setting remained depressive symptomfree compared to patients from the acute care setting ( $78 \%$ vs. $45 \%, p<.001$ ). In contrast, a higher percentage of patients from the acute care setting experienced improved depressive symptoms, developing depressive symptoms, or persistent depressive symptoms when compared to patients from the outpatient setting (Figure 1).

Table 3 shows the mean levels of depressive symptoms and the average change in PHQ-9 scores for patients who were depressive symptom-free, whose depressive symptoms improved, whose depressive symptoms developed, or who had persistent depressive symptoms. There was a significant group by time interaction (Wilks' Lamda $=.53, \mathrm{~F}=74$, $\mathrm{df}=3, p<.001$, partial eta squared $=.47$ ), but no significant time effect. Figure 2 depicts the median, minimum, maximum, first quartile, and third quartile depressive symptom scores for each of the four groups at baseline and three or six months.

\section{Depressive symptom change and HRQOL}

The Minnesota Living with HF mean scores for the entire sample were $43 \pm 27$ at baseline, $36 \pm 26$ at 3-6 months, and $36 \pm 25$ at one year. When we assessed the HRQOL trajectory between the four groups (depressive symptom-free, depressive symptoms improved, depressive symptoms developed, and persistent depressive symptoms), there was a 
significant interaction between group and time (Wilks' Lambda $=.82, \mathrm{~F}=8.2, \mathrm{df}=6, p<$. 001, partial eta squared $=.10$ ), as well as a significant main effect for time (Wilks' Lambda $=.93, \mathrm{~F}=8.9, \mathrm{df}=2, p<.001$, partial eta squared $=.07$ ). Patients who were depressive symptom-free had the best HRQOL at all three time points, while patients with persistent depressive symptoms had the worst HRQOL. Figure 3 displays the median, minimum, maximum, first quartile, and third quartile, and outlier HRQOL scores for each of the four groups. The results in this figure show that as patients' depressive symptoms improved or developed from baseline to 3-6 months, they experienced a corresponding change in HRQOL.

Figure 4 shows the difference in HRQOL scores at one year between the four groups $(\mathrm{F}=36.1, \mathrm{df}=3, p<.001)$. Post-hoc comparisons indicated that patients who remained symptom-free had the best HRQOL at one year $(27.2 \pm 21, p<.01)$ compared to patients whose depressive symptoms improved (40.4 \pm 24$)$, depressive symptoms developed (56.1 \pm 13.6), or had persistent depressive symptoms $(64 \pm 22.1)$. The patients whose depressive symptoms improved had better HRQOL than patients with persistent depressive symptoms $(\mathrm{p}<.001)$. There was a trend for patients whose depressive symptoms improved to have better HRQOL at one year compared to patients who developed depressive symptoms ( $p=$. $09)$. There was no difference between patients who developed depressive symptoms and those with persistent depressive symptoms $(p=.6)$; both groups had equally poor HRQOL at one year.

In the multiple regression analysis (Table 4), depressive symptom trajectory from baseline to 3-6 months was a significant predictor of HRQOL at one year, controlling for age, gender, ejection fraction, recruitment setting, treatment group, NYHA functional class, and DASI score. The model explained 39\% of the variance in HRQOL; depressive symptom status by itself explained $14 \%$ of the variance. Younger age and lower scores on the DASI at baseline were also significant predictors of worse HRQOL at one year. However, depressive symptom status had the largest standardized beta value $(\beta=.42)$, indicating that depressive symptom status was the strongest predictor of worse HRQOL after controlling for all other variables in the model.

\section{Discussion}

Although depressive symptoms have been described as an independent predictor of HRQOL, our knowledge was previously limited by primarily cross-sectional study designs. Our study has advanced the state of the science on depression in patients with HF by demonstrating that a change in depressive symptoms over time predicts future HRQOL. We found that patients with $\mathrm{HF}$ who remain depressive symptom-free or whose depressive symptoms improve have substantially better HRQOL at one year than patients with persistent depressive symptoms.

This study is one of few to examine the trajectory of depressive symptoms in patients with HF. ${ }^{11-14}$ We found that half of patients with depressive symptoms at baseline experienced a clinically significant improvement in depressive symptoms by 3 to 6 months, while the other half had persistent depressive symptoms. Furthermore, a small percentage of patients who were depressive symptom-free at baseline had a clinically significant increase in depressive symptoms by 3 to 6 months. These findings are consistent with results from other studies. Koenig et al. ${ }^{14}$ followed elderly hospitalized patients with HF and found that approximately half of those with a major or minor depressive disorder experienced remission within one year. In two additional studies, Koenig et al. followed elderly hospitalized patients with HF and chronic pulmonary disease to determine the trajectory of minor ${ }^{12}$ and major depression ${ }^{11}$ as measured with the Structured Clinical Interview. Among patients with major 
depression, $56 \%$ experienced remission within six months after hospitalization. Similarly, $65 \%$ of patients with minor depression improved within three months. Fulop et al. ${ }^{13}$ described a similar course of depressive symptoms in elderly hospitalized patients with HF. At six months, $29 \%$ of patients with depressive symptoms at baseline continued to have persistent depressive symptoms; while $6 \%$ of patients who were non-depressed at baseline developed depressive symptoms. Our results, combined with findings from these investigators, suggest that a significant portion of outpatients and inpatients with HF experience persistent depressive symptoms, while other patients experience fluctuations in depressive symptoms.

The baseline HRQOL level among patients in our study, measured with the Minnesota Living with HF questionnaire, is similar to that of nine studies described by Riegel et al. ${ }^{17}$ The HRQOL scores for patients in our study improved substantially from baseline to the 3-6 month assessment and then stabilized. This finding is supported by results from Moser et al., ${ }^{5}$ who reported that HRQOL scores, as measured with the Minnesota Living with HF questionnaire, rapidly improve within the first month after a HF hospitalization and then become constant.

A novel finding from our study was that the trajectories of depressive symptoms and HRQOL were closely linked over time. We found that as patients' depressive symptoms improved, worsened, or stayed the same, there was a parallel change in HRQOL. Other researchers examining the relationship between depressive symptoms and HRQOL in patients with $\mathrm{HF}$ in cross-sectional studies have identified depressive symptoms as an independent predictor of HRQOL. ${ }^{2,7-10}$ Similar to our study, these investigators measured HRQOL using HF-specific measures - either the Minnesota Living with HF questionnaire or the Kansas City Cardiomyopathy questionnaire. In one cross-sectional study, Faller et al. ${ }^{32}$ evaluated depressive symptoms, NYHA functional status, and HRQOL in outpatients with HF. The investigators found that among patients with depressive symptoms, there was no dose-response relationship between worsening functional status and the life satisfaction component of HRQOL. Their results suggest that depressive symptoms may be more important than functional status as a determinant of HRQOL.

However, it is not possible to draw conclusions from these cross-sectional studies regarding the sequential relationship between depressive symptoms and HRQOL. In our longitudinal study, we provided evidence that the depressive symptom trajectory from baseline to 3-6 months predicts HRQOL at one year. The closely aligned trajectories of depressive symptoms and HRQOL suggest a bi-directional relationship between these two variables. This finding is supported by results from Evangelista et al., 33 who followed patients with advanced HF referred for transplantation. In this study, HRQOL was measured with a generic measure of HRQOL - the Short-Form 12. Depressive symptoms and HRQOL were assessed at baseline and two years. The researchers found that the patients who stabilized medically and were taken off the transplant list had better HRQOL and lower levels of depressive symptoms at two years when compared to patients who remained on the transplant list or received a transplant.

In our study, we were able to control for subjective functional status as measured by both NYHA functional classification and the Duke Activity Status Index (DASI). Both depressive symptom status and DASI scores were significant predictors of HRQOL at one year, but depressive symptom status was the strongest independent predictor. Due to data limitations, we were not able to explore the impact of changes in functional status on subsequent HRQOL. As functional status is an important component of the conceptual framework of HRQOL, ${ }^{15}$ future researchers should obtain frequent, serial measures of 
functional status, depressive symptoms, and HRQOL in order to tease out any possible cause-effect relationships in this population.

Previous investigators have hypothesized that interventions aimed at reducing depressive symptoms in patients with HF may also improve patients' HRQOL. Although the state of the science is not well developed in this area, results from three trials support this hypothesis. Gary et al. ${ }^{34}$ tested the effects of a home-based exercise program in elderly female outpatients with diastolic HF. The exercise group experienced a greater improvement in both depressive symptoms and HF-specific HRQOL when compared to a group who received education only. Gary et al. ${ }^{35}$ also compared usual care with home-based exercise, cognitive behavioral therapy, and combined exercise and cognitive behavioral therapy for the treatment of major and minor depression in outpatients with HF. The investigators found no significant differences over time in depressive symptoms and HF-specific HRQOL between the four groups. However, when data from patients with major depression were analyzed separately, the researchers found that only the combined exercise and cognitive behavioral therapy group demonstrated a sustained decrease in depression scores at 12 and 24 weeks. Furthermore, the patients with major depression in the combined intervention group experienced the greatest improvement in HRQOL. Sullivan et al. ${ }^{36}$ provided further support for the hypothesis that interventions aimed at reducing depressive symptoms can improve HF-specific HRQOL. In a prospective cohort study, the investigators assigned outpatients with HF to group mindfulness-based intervention or usual care control. Patients in the intervention group experienced significant improvements in both depressive symptoms and HRQOL when compared to usual care.

Although investigators have provided some evidence that the reduction of depressive symptoms may lead to improved HRQOL in patients with HF, further research is needed. The intervention studies discussed above enrolled patients from the outpatient setting, thus, it is not known whether a psychological intervention would be beneficial for patients in the acute care setting. Furthermore, the appropriate intensity or dose of intervention needed to improve depressive symptoms and HRQOL is not known. An intriguing study conducted by Powell et al. ${ }^{37}$ demonstrated that a single, brief counseling session improved depression and HRQOL among women recently diagnosed with cancer. It is possible that a brief psychosocial intervention for depressive symptoms may be appropriate for patients with $\mathrm{HF}$ in the acute care setting. To further explore this treatment option, our team is currently conducting a randomized, controlled trial testing the effects of a nurse-delivered, brief cognitive therapy intervention on depressive symptoms and HRQOL in hospitalized patients with HF (Clinicaltrials.gov identifier: NCT01275742).

\section{Limitations}

We included patients enrolled from two distinct HF populations-acute care and outpatient. This approach however, can be considered strength because it improved the generalizability of our findings and demonstrated that the relationship between depressive symptoms and HRQOL holds across different HF patient populations. Data on medications were not collected at one study site. Therefore, we were unable to explore the impact of HF medications or antidepressants on HRQOL. Future research on the relationship between the trajectory of depressive symptoms and HRQOL should include the effects of antidepressants and other medications that may affect HRQOL.

\section{Conclusions}

In this study, we demonstrated that a change in depressive symptoms over time predicts subsequent HRQOL in patients with HF. We found that patients who remain depressive symptom-free or whose symptoms improve have substantially better HRQOL at one year 
compared to patients with persistent depressive symptoms. Depressive symptoms are a significant clinical problem in patients with HF. Given that patients with HF may experience persistent depressive symptoms or develop depressive symptoms that predict worsened HRQOL, it is important for clinicians to regularly assess for depressive symptoms in this population. In addition, researchers should continue to test interventions to reduce depressive symptoms in patients with HF. In particular, research is needed to determine whether the acute care setting is an appropriate time and place to introduce such interventions. Our findings suggest that the trajectories of depressive symptoms and HRQOL are closely related. Future researchers are urged to include HRQOL as a primary outcome when testing the effects of treatments for depressive symptoms in patients with HF.

\section{Acknowledgments}

The authors would like to acknowledge Susan M. Westneat, MA, for her assistance with data management.

\section{Funding Sources}

This research was funded by the National Institutes of Health, National Institute of Nursing Research 5R01 NR 008567, NINR P20 NR010679, and NIH M01 RR02602. The content is solely the responsibility of the authors and does not necessarily represent the official views of the National Institute of Nursing Research or the National Institutes of Health.

\section{References}

1. Rutledge T, Reis VA, Linke SE, Greenberg BH, Mills PJ. Depression in heart failure: a metaanalytic review of prevalence, intervention effects, and associations with clinical outcomes. J Am Coll Cardiol. 2006 Oct.48:1527-1537. [PubMed: 17045884]

2. Gottlieb SS, Khatta M, Friedmann E, Einbinder L, Katzen S, Baker B, et al. The influence of age, gender, and race on the prevalence of depression in heart failure patients. J Am Coll Cardiol. 2004 May.43:1542-1549. [PubMed: 15120809]

3. Omery, AK.; Dean, H. Multiple instruments for measuring quality of life. In: Frank-Stromborg, M.; Olsen, SJ., editors. Instruments for Clinical Health-Care Research. 3rd ed.. Boston: Jones and Bartlett Publishers; 2004. p. 150-163.

4. Konstam V, Salem D, Pouleur H, Kostis J, Gorkin L, Shumaker S, et al. Baseline quality of life as a predictor of mortality and hospitalization in 5,025 patients with congestive heart failure. Am J Cardiol. 1996 Oct.78:890-895. [PubMed: 8888661]

5. Moser DK, Yamokoski L, Sun JL, Conway GA, Hartman KA, Graziano JA, et al. Improvement in health-related quality of life after hospitalization predicts event-free survival in patients with advanced heart failure. J Card Fail. 2009 Nov.15:763-769. [PubMed: 19879462]

6. Stanek EJ, Oates MB, McGhan WF, Denofrio D, Loh E. Preferences for treatment outcomes in patients with heart failure: symptoms versus survival. J Card Fail. 2000 Sep.6:225-232. [PubMed: 10997749]

7. Hallas CN, Wray J, Andreou P, Banner NR. Depression and perceptions about heart failure predict quality of life in patients with advanced heart failure. Heart Lung. 2010 Apr. [Epub ahead of print].

8. Faller H, Stork S, Schuler M, Schowalter M, Steinbuchel T, Ertl G, et al. Depression and disease severity as predictors of health-related quality of life in patients with chronic heart failure--a structural equation modeling approach. J Card Fail. 2009 May.15:286-292. [PubMed: 19398075]

9. Pressler SJ, Subramanian U, Kareken D, Perkins SM, Gradus-Pizlo I, Sauve MJ, et al. Cognitive deficits and health-related quality of life in chronic heart failure. J Cardiovasc Nurs. 2010 May-Jun. 25:189-198. [PubMed: 20357665]

10. Ancheta IB, Battie C, Cobb S, Ancheta C, Miller A, Chiong JR. The impact of B-type natriuretic peptide, New York Heart Association classification and depression on quality of life in nonhospitalized heart failure patients. Prog Cardiovasc Nurs. 2009 Dec.24:124-130. [PubMed: 20002336] 
11. Koenig HG, Johnson JL, Peterson BL. Major depression and physical illness trajectories in heart failure and pulmonary disease. J Nerv Ment Dis. 2006 Dec.194:909-916. [PubMed: 17164629]

12. Koenig HG, Vandermeer J, Chambers A, Burr-Crutchfield L, Johnson JL. Minor depression and physical outcome trajectories in heart failure and pulmonary disease. J Nerv Ment Dis. 2006 Mar. 194:209-217. [PubMed: 16534439]

13. Fulop G, Strain JJ, Stettin G. Congestive heart failure and depression in older adults: clinical course and health services use 6 months after hospitalization. Psychosomatics. 2003 Sep-Oct. 44:367-373. [PubMed: 12954910]

14. Koenig HG. Depression in hospitalized older patients with congestive heart failure. Gen Hosp Psychiatry. 1998 Jan.20:29-43. [PubMed: 9506252]

15. Kop WJ, Synowski SJ, Gottlieb SS. Depression in heart failure: biobehavioral mechanisms. Heart Fail Clin. 2010 Jan.7:23-38. [PubMed: 21109205]

16. Riegel B, Moser DK, Rayens MK, Carlson B, Pressler SJ, Shively M, et al. Ethnic differences in quality of life in persons with heart failure. J Card Fail. 2008 Feb.14:41-47. [PubMed: 18226772]

17. Riegel B, Moser DK, Glaser D, Carlson B, Deaton C, Armola R, et al. The Minnesota Living With Heart Failure Questionnaire: sensitivity to differences and responsiveness to intervention intensity in a clinical population. Nurs Res. 2002 Jul-Aug.51:209-218. [PubMed: 12131233]

18. Judd LL, Akiskal HS, Zeller PJ, Paulus M, Leon AC, Maser JD, et al. Psychosocial disability during the long-term course of unipolar major depressive disorder. Arch Gen Psychiatry. 2000 Apr.57:375-380. [PubMed: 10768699]

19. Saveanu RV, Mayes T. Diagnosing depression in congestive heart failure. Heart Fail Clin. Jan. 7:75-79. [PubMed: 21109210]

20. Williams JW Jr, Noel PH, Cordes JA, Ramirez G, Pignone M. Is this patient clinically depressed? JAMA. 2002 Mar.287:1160-1170. [PubMed: 11879114]

21. Koenig HG, George LK, Peterson BL, Pieper CF. Depression in medically ill hospitalized older adults: prevalence, characteristics, and course of symptoms according to six diagnostic schemes. Am J Psychiatry. 1997 Oct.154:1376-1383. [PubMed: 9326819]

22. Gilbody S, Richards D, Brealey S, Hewitt C. Screening for depression in medical settings with the Patient Health Questionnaire (PHQ): a diagnostic meta-analysis. J Gen Intern Med. 2007 Nov. 22:1596-1602. [PubMed: 17874169]

23. Chung ML, Lennie TA, De Jong M, Wu JR, Riegel B, Moser DK. Patients differ in their ability to self-monitor adherence to a low-sodium diet versus medication. J Card Fail. 2008 Mar.14:114120. [PubMed: 18325457]

24. Lowe B, Kroenke K, Herzog W, Grafe K. Measuring depression outcome with a brief self-report instrument: sensitivity to change of the Patient Health Questionnaire (PHQ-9). J Affect Disord. 2004 Jul.81:61-66. [PubMed: 15183601]

25. Pressler SJ, Subramanian U, Perkins SM, Gradus-Pizlo I, Kareken D, Kim J, et al. Measuring depressive symptoms in heart failure: validity and reliability of the Patient Health Questionnaire-8. Am J Crit Care. 2010 Mar.20:146-152. [PubMed: 20378777]

26. Rector TS, Kubo SH, Cohn JN. Patient's self-assessment of their congestive heart failure: Content, reliability and validity of a new measure, the Minnesota Living with Heart Failure Questionnaire. Heart Failure. 1987; 1:198-209.

27. Heo S, Moser DK, Riegel B, Hall LA, Christman N. Testing the psychometric properties of the Minnesota Living with Heart Failure questionnaire. Nurs Res. 2005 Jul-Aug.54:265-272. [PubMed: 16027569]

28. Hlatky MA, Boineau RE, Higginbotham MB, Lee KL, Mark DB, Califf RM, et al. A brief selfadministered questionnaire to determine functional capacity (the Duke Activity Status Index). Am J Cardiol. 1989 Sep.64:651-654. [PubMed: 2782256]

29. Mills RM Jr, Haught WH. Evaluation of heart failure patients: objective parameters to assess functional capacity. Clin Cardiol. 1996 Jun.19:455-460. [PubMed: 8790948]

30. Katz JN, Chang LC, Sangha O, Fossel AH, Bates DW. Can comorbidity be measured by questionnaire rather than medical record review? Med Care. 1996 Jan.34:73-84. [PubMed: 8551813] 
31. Charlson ME, Pompei P, Ales KL, MacKenzie CR. A new method of classifying prognostic comorbidity in longitudinal studies: development and validation. J Chronic Dis. 1987; 40:373383. [PubMed: 3558716]

32. Faller H, Steinbuchel T, Stork S, Schowalter M, Ertl G, Angermann CE. Impact of depression on quality of life assessment in heart failure. Int J Cardiol. 2010 Jul.142:133-137. [PubMed: 19162345]

33. Evangelista LS, Dracup K, Moser DK, Westlake C, Erickson V, Hamilton MA, et al. Two-year follow-up of quality of life in patients referred for heart transplant. Heart Lung. 2005 May-Jun. 34:187-193. [PubMed: 16015223]

34. Gary RA, Sueta CA, Dougherty M, Rosenberg B, Cheek D, Preisser J, et al. Home-based exercise improves functional performance and quality of life in women with diastolic heart failure. Heart Lung. 2004 Jul-Aug.33:210-218. [PubMed: 15252410]

35. Gary RA, Dunbar SB, Higgins MK, Musselman DL, Smith AL. Combined exercise and cognitive behavioral therapy improves outcomes in patients with heart failure. J Psychosom Res. 2010 Aug. 69:119-131. [PubMed: 20624510]

36. Sullivan MJ, Wood L, Terry J, Brantley J, Charles A, McGee V, et al. The Support, Education, and Research in Chronic Heart Failure Study (SEARCH): a mindfulness-based psychoeducational intervention improves depression and clinical symptoms in patients with chronic heart failure. Am Heart J. 2009 Jan.157:84-90. [PubMed: 19081401]

37. Powell CB, Kneier A, Chen LM, Rubin M, Kronewetter C, Levine E. A randomized study of the effectiveness of a brief psychosocial intervention for women attending a gynecologic cancer clinic. Gynecol Oncol. 2008 Oct.111:137-143. [PubMed: 18703220] 


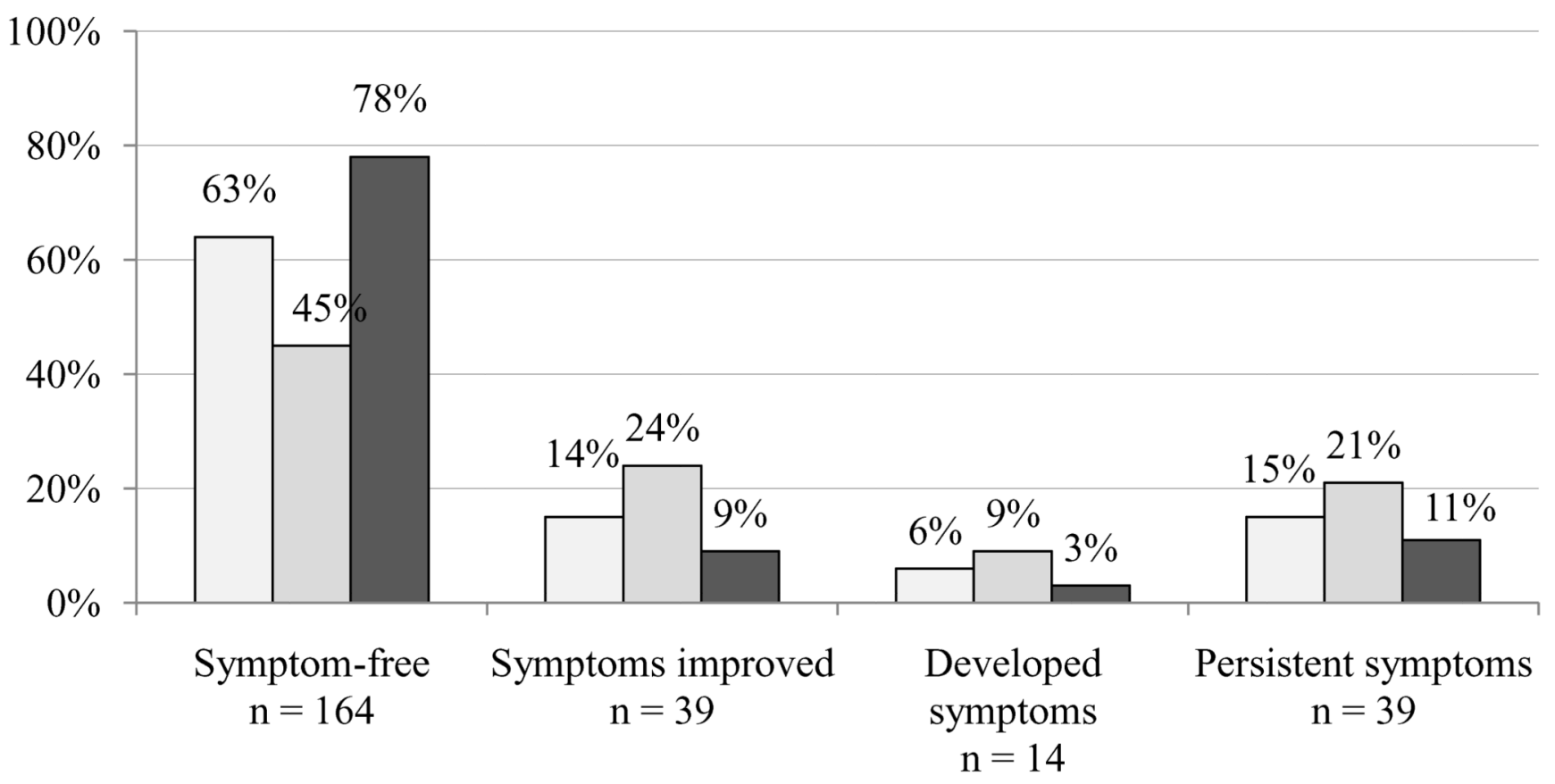

Figure 1.

Depressive symptom status change from baseline to 3-6 months for the overall sample and two study sites*

*Chi-square, $p<.001$ 


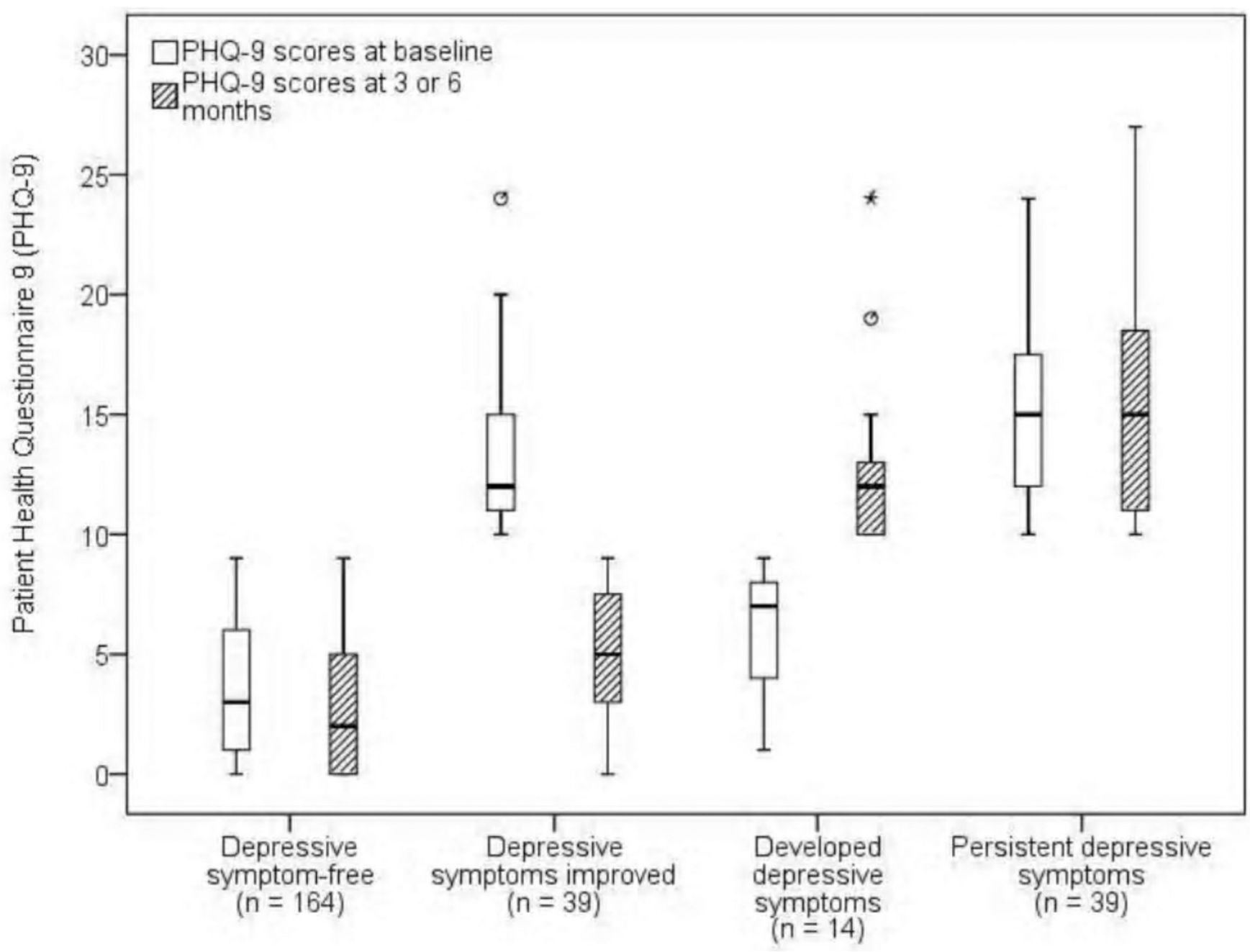

Figure 2.

Box plot with median levels of depressive symptoms in patients with HF at baseline and three or six months*

$*$ Median $=$ horizontal line, first quartile $=$ bottom of box, third quartile $=$ top of box, vertical line $=$ minimum and maximum, ${ }^{*}$ or ${ }^{\circ}$ indicate patient outliers 


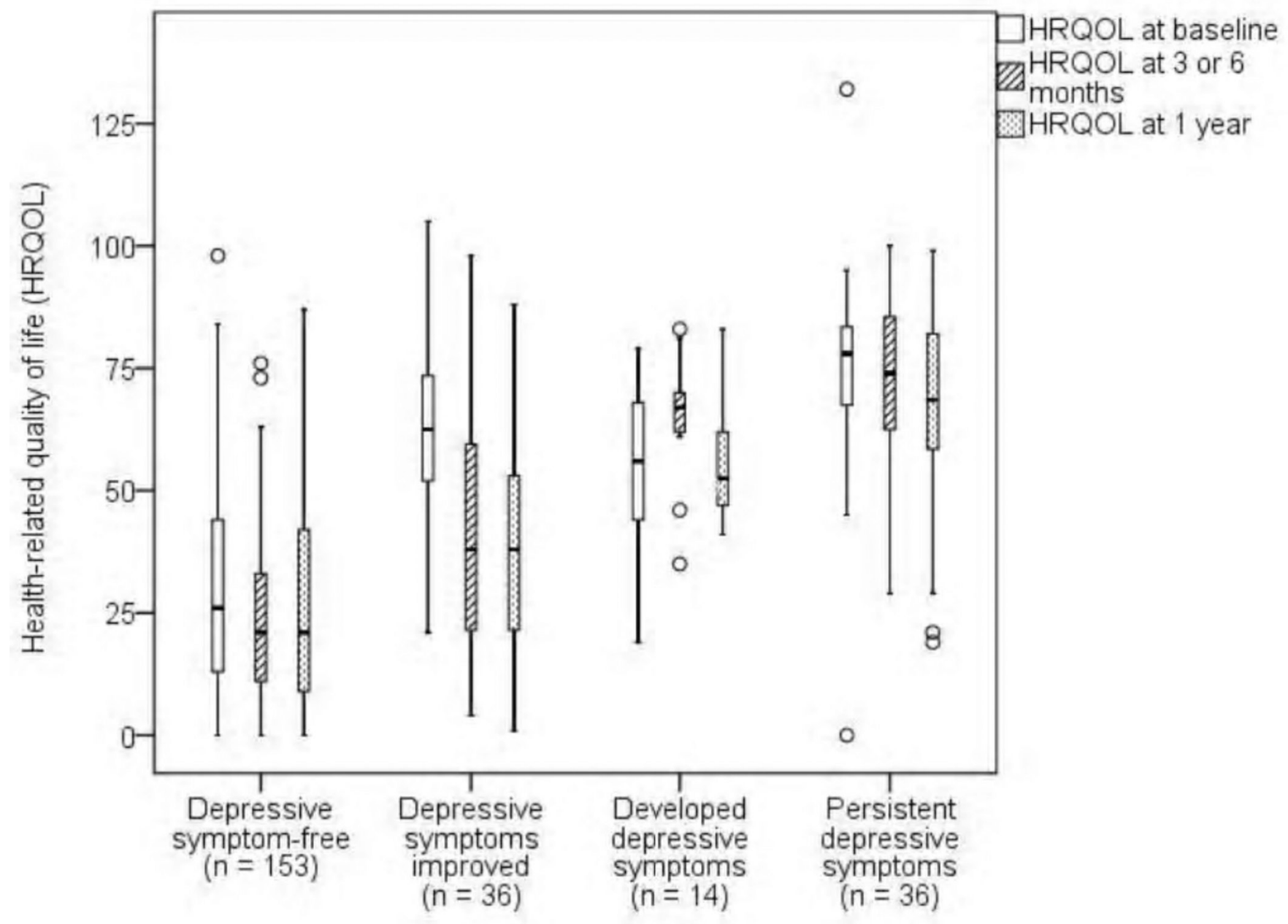

Figure 3.

Box plot with median levels of health-related quality of life in patients with HF at baseline, three or six months, and one year*

*Higher scores mean worse health-related quality of life (HRQOL); Median = horizontal line, first quartile $=$ bottom of box, third quartile $=$ top of box, vertical line $=$ minimum and maximum, ${ }^{\circ}$ indicates patient outliers. 


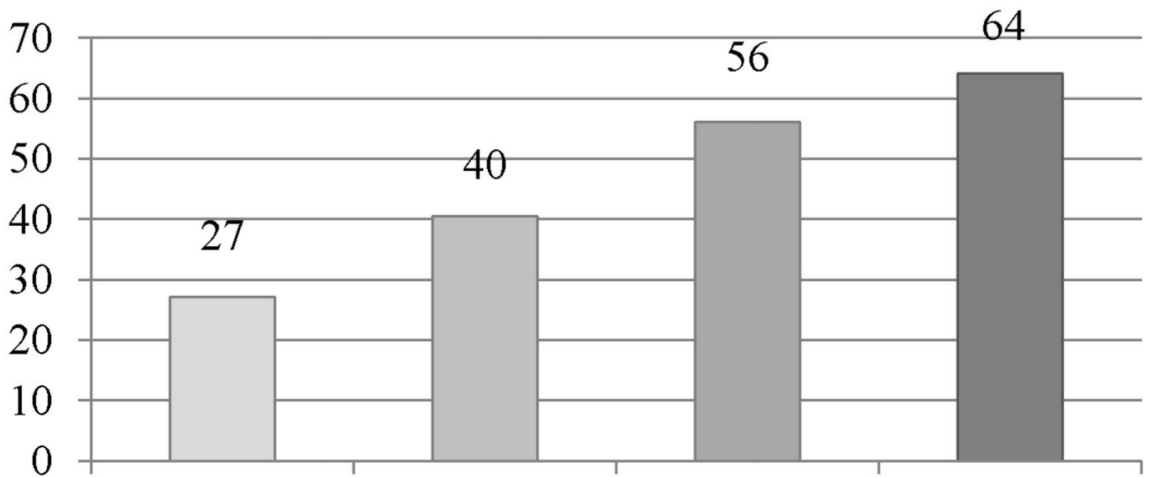

(A)

Depressive symptom-free

$(n=164)$
(C) Developed depressive symptoms ( $\mathrm{n}=14)$
(D)

Persistent depressive symptoms $(\mathrm{n}=39)$
(B)

Depressive symptoms improved $(\mathrm{n}=39)$
Tukey HSD posthoc tests

\begin{tabular}{lc}
\hline $\mathrm{A}<\mathrm{B}$ & $p=.004$ \\
$\mathrm{~A}<\mathrm{C}$ & $p<.001$ \\
$\mathrm{~A}<\mathrm{D}$ & $p<.001$ \\
$\mathrm{~B}=\mathrm{C}$ & $p=.09$ \\
$\mathrm{~B}<\mathrm{D}$ & $p<.001$ \\
$\mathrm{C}=\mathrm{D}$ & $p=.6$ \\
\hline
\end{tabular}

Figure 4.

Comparison of HRQOL* at one year between patients with HF categorized into four groups by change in depressive symptoms from baseline to 3-6 months *Higher scores mean worse health-related quality of life (HRQOL); one-way analysis of variance; $\mathrm{F}=36.1, \mathrm{df}=3, p<.001$ 
Table 1

Depressive symptom status group assignment based on PHQ-9 scores at baseline and 3 or 6 months.

\begin{tabular}{ccl}
\hline $\begin{array}{c}\text { PHQ-9 at } \\
\text { baseline }\end{array}$ & $\begin{array}{c}\text { PHQ-9 at } \\
\text { 3 or } 6 \text { months }\end{array}$ & $\begin{array}{l}\text { Depressive symptom status group } \\
\text { assignment }\end{array}$ \\
\hline $0-9$ & $0-9$ & Depressive symptom-free \\
\hline$\geq 10$ & $0-9$ & Depressive symptoms improved \\
\hline $0-9$ & $\geq 10$ & Depressive symptoms developed \\
\hline$\geq 10$ & $\geq 10$ & Persistent depressive symptoms \\
\hline
\end{tabular}

PHQ-9 = Patient Health Questionnaire-9 
Table 2

Baseline characteristics for the total sample and comparison of patient characteristics* from study site 1 (acute care setting; Ohio) and site 2 (outpatient setting; Kentucky)

\begin{tabular}{|c|c|c|c|c|}
\hline Characteristic & $\begin{array}{c}\text { Overall } \\
(\mathrm{n}=\mathbf{2 5 6})\end{array}$ & $\begin{array}{c}\text { Study Site } 1 \\
(\mathrm{n}=108)\end{array}$ & $\begin{array}{c}\text { Study Site } 2 \\
(\mathrm{n}=148)\end{array}$ & $p$ \\
\hline Age, mean $\pm S D$, yrs & $61.2 \pm 11.9$ & $62.7 \pm 11.5$ & $60 \pm 12.2$ & .07 \\
\hline Female, $\%$ & 35.1 & 37.7 & 33.1 & .3 \\
\hline Minority, \% & 18.1 & 15.8 & 19.9 & .3 \\
\hline Lives alone, $\%$ & 23.5 & 21.9 & 24.7 & .4 \\
\hline Married, \% & 58.5 & 63.2 & 56.1 & .2 \\
\hline $\mathrm{LVEF}$, mean $\pm \mathrm{SD}, \%$ & $32.4 \pm 14.5$ & $26.8 \pm 12.8$ & $37 \pm 14.2$ & $<.001$ \\
\hline Body mass index, mean $\pm \mathrm{SD}$ & $31.2 \pm 8.1$ & $29.5 \pm 7.1$ & $32.5 \pm 8.6$ & .003 \\
\hline \multicolumn{5}{|l|}{ NYHA functional class, $\%$} \\
\hline Class I & 7.2 & 4.4 & 9.3 & \\
\hline Class II & 38.6 & 28.3 & 43.4 & \\
\hline Class III & 48.5 & 61.1 & 39.1 & \\
\hline Class IV & 5.7 & 6.2 & 5.3 & .003 \\
\hline Duke Activity Status Index & $14.2 \pm 13.6$ & $10 \pm 10$ & $17.4 \pm 15$ & $<.001$ \\
\hline Education, mean $\pm \mathrm{SD}$, yrs & $13.6 \pm 3.3$ & $13.1 \pm 3$ & $13.9 \pm 3.4$ & .03 \\
\hline \multicolumn{5}{|l|}{ Heart failure etiology, $\%$} \\
\hline Ischemic & 46 & 49.1 & 43.7 & \\
\hline Idiopathic & 22.6 & 21.1 & 23.8 & \\
\hline Other & 31.3 & 29.8 & 32.5 & .7 \\
\hline \multicolumn{5}{|l|}{ Comorbidity, $\%$} \\
\hline History of MI & 48.7 & 50.9 & 47.3 & .3 \\
\hline History of CABG & 31.3 & 41.2 & 23.8 & .003 \\
\hline Atrial fibrillation & 48.7 & 55.3 & 43.7 & .06 \\
\hline Biventricular pacemaker & 31.7 & 26.3 & 35.8 & .1 \\
\hline ICD & 39.2 & 37.7 & 40.4 & .7 \\
\hline History of stroke & 18.1 & 17.5 & 18.5 & .5 \\
\hline Diabetes mellitus & 39.6 & 36 & 42.4 & .3 \\
\hline COPD & 18.5 & 21.9 & 16 & .2 \\
\hline
\end{tabular}

Baseline medication, $\%^{\dagger}$

\begin{tabular}{lcccc} 
ACE inhibitor & - & - & 70.9 & - \\
Beta-blocker & - & - & 86.1 & - \\
Diuretic & - & - & 67.5 & - \\
Digoxin & - & - & 19.9 & - \\
ARB & - & - & 13.9 & - \\
Antidepressants & - & - & 22.7 & - \\
\hline
\end{tabular}

${ }^{*} p$ values were calculated using two-sample $t$ tests for continuous variables and the chi-square test of association for categorical variables

${ }^{\dagger}$ Data on medications were not available at one study site

J Card Fail. Author manuscript; available in PMC 2012 September 1. 
$\mathrm{LVEF}=$ Left ventricular ejection fraction, $\mathrm{NYHA}=\mathrm{New}$ York Heart Association functional class, $\mathrm{MI}=$ myocardial infarction, $\mathrm{CABG}=$ coronary artery bypass graft, $\mathrm{ICD}=$ implanted cardiac defibrillator, $\mathrm{COPD}=$ chronic obstructive pulmonary disease, $\mathrm{ACE}=$ angiotensin converting enzyme, $\mathrm{ARB}=$ angiotensin receptor blocker 
Table 3

Depressive symptom scores of the four groups at baseline and 3 or 6 months

\begin{tabular}{lccc}
\hline Depressive symptom status group & $\begin{array}{c}\text { Baseline PHQ-9 } \\
\text { Mean } \pm \text { SD }\end{array}$ & $\begin{array}{c}\text { PHQ-9 at 3 or 6 } \\
\text { months } \\
\text { Mean } \pm \text { SD }\end{array}$ & $\begin{array}{c}\text { Average change } \\
\text { in PHQ-9 score } \\
\text { Mean } \pm \text { SD }\end{array}$ \\
\hline Depressive symptom-free $(\mathrm{n}=164)$ & $3.6 \pm 2.9$ & $2.9 \pm 2.8$ & $0.7 \pm 3.2$ \\
\hline Depressive symptoms improved $(\mathrm{n}=39)$ & $13.7 \pm 3.5$ & $5.2 \pm 2.7$ & $8.5 \pm 4.5$ \\
\hline Depressive symptoms developed $(\mathrm{n}=14)$ & $6.1 \pm 2.5$ & $13 \pm 4.0$ & $-6.9 \pm 4.8$ \\
\hline Persistent depressive symptoms $(\mathrm{n}=39)$ & $15.1 \pm 3.6$ & $15.4 \pm 4.6$ & $-0.3 \pm 4.6$ \\
\hline
\end{tabular}

PHQ-9 = Patient Health Questionnaire-9; SD = standard deviation 


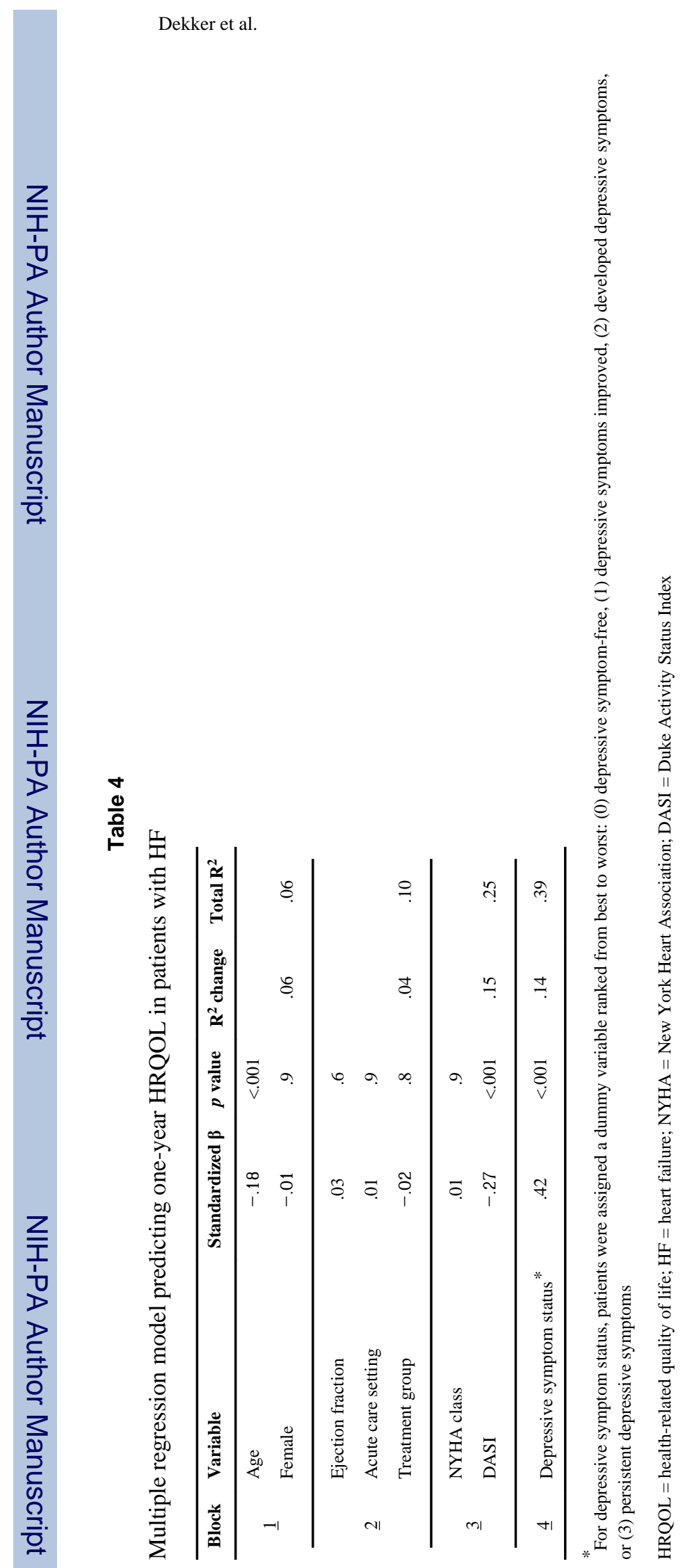

J Card Fail. Author manuscript; available in PMC 2012 September 1. 